\title{
Serotonin Syndrome Presenting as a Posterior Reversible Encephalopathy Syndrome
}

\author{
Muhammad Taimur Malik Mohammad Faraz Majeed Ramin Zand \\ Geisinger Health System, Danville, PA, USA
}

\section{Keywords}

Posterior reversible encephalopathy syndrome $\cdot$ Serotonin syndrome $\cdot$ Venlafaxine $\cdot$ Cerebral vasoconstriction $\cdot$ Adverse reactions

\begin{abstract}
Posterior reversible encephalopathy syndrome (PRES) is a neurological syndrome characterized by an altered level of consciousness, headaches, seizure, and visual changes. PRES has several different etiologies, including malignant hypertension, eclampsia, and certain medications. Here, we describe a 41-year-old woman who presented with altered mental status. She had a preliminary diagnosis of serotonin syndrome as she was on many different serotoninsparing agents, but her imaging findings were consistent with PRES. After her medications were reviewed and the causative agent was removed, the patient's neurological exam and imaging findings improved, and she returned to her baseline. To our knowledge, this is a unique case of PRES caused by serotonin syndrome secondary to venlafaxine usage.
\end{abstract}

(C) 2020 The Author(s)

Published by S. Karger AG, Basel

\section{Introduction}

Posterior reversible encephalopathy syndrome (PRES), also known as reversible posterior leukoencephalopathy syndrome, is a unique entity of neurological and radiological findings which is characterized by an altered level of consciousness, seizures, visual changes, and 
Malik et al.: Serotonin Syndrome Presenting as a Posterior Reversible Encephalopathy Syndrome

headache [1]. The diagnosis is usually based on clinical and radiological biomarkers. The most common etiology of PRES is hypertension, followed by immunosuppressive medications, and eclampsia [2-4]. The exact pathogenesis of PRES is still not known, but it is thought to be associated with loss of cerebral autoregulation and endothelial dysfunction [3]. Treatment includes correcting the blood pressure and removing the offending agent. Here, we describe a patient who initially presented with altered mental status. She had a preliminary diagnosis of serotonin syndrome, and her imaging findings were consistent with PRES.

\section{Case Description}

A 41-year-old woman was transferred to the emergency room at an outside hospital $2 \mathrm{~h}$ after finishing her dialysis with altered mental status, combativeness, nausea, and vomiting. She was stabilized and intubated for airway protection in the emergency room. She was then transferred to the intensive care unit at our hospital for a higher level of care. Prior to this event, the patient was in her normal state of health, except for some mild headache that was relieved by ibuprofen. Her past medical history was significant for type 1 diabetes, end-stage renal disease on hemodialysis, gastroesophageal reflux disease, and bipolar disorder. Her home medications included venlafaxine, buspirone, simvastatin, aspirin, omeprazole, lisinopril, insulin glargine, and insulin aspart. There was no prior history of smoking, alcohol, or illicit drug use. Her vitals showed a blood pressure of 188/80 mm Hg, pulse of 127/min, temperature of $101.3 \mathrm{~F}$, and $\mathrm{O}_{2}$ saturation of $98 \%$ on ventilator. Neurological examination was significant for increased muscle tone in lower extremities with symmetrically increased deep tendon reflexes. Higher mental functions could not be assessed as patient was on sedative medications. There was no sign of meningismus. Initial laboratory data showed a mild leukocytosis, elevated creatinine, elevated lactic acid, and negative urine toxicology screen. The initial head CT in the emergency room was unremarkable. Based on patient's presentation, there was concern for possible meningoencephalitis; therefore, she was started on broad-spectrum antibiotic coverage with cefepime, vancomycin, and acyclovir. Blood cultures, urine culture, cerebrospinal fluid (CSF) for Gram stain and culture were obtained on day 1 prior to antibiotic administration. The CSF results including bacterial culture and viral polymerase chain reaction were negative, and antibiotics were discontinued. An electroencephalogram was performed on day 1 which showed diffuse slowing, consistent with encephalopathy. Magnetic resonance imaging (MRI) of the brain on day 1 revealed restricted diffusion and apparent diffusion coefficient (ADC) hyperintensity involving the right parietal and temporal lobes (Fig. 1). A magnetic resonance angiography (MRA) of the head and neck performed on day 2 did not show any acute stenosis that would be concerning for cerebral vasospasm and reversible cerebral vasoconstriction syndrome (RCVS).

After careful review of patient's MRI brain, ADC was bright consistent with vasogenic edema that goes more along with PRES. On review of the patient's medications, she was prediagnosed with serotonin syndrome since she was on many different serotonergic agents. After her venlafaxine and buspirone were discontinued on day 3, the patient's altered mental status resolved, and she was extubated. Her tremor and hypertonia improved with cyproheptadine and baclofen around day 5. A repeat brain MRI 1 week later showed interval resolution in the restricted diffusion from prior MRI (Fig. 2). The patient was at her previous baseline neurological function when we evaluated her in our outpatient clinic after 1 month. 
Malik et al.: Serotonin Syndrome Presenting as a Posterior Reversible Encephalopathy Syndrome

\section{Discussion}

Selective serotonin and norepinephrine reuptake inhibitors or selective serotonin reuptake inhibitors (SSRIs) when combined with other serotonergic agents such as buspirone, triptans, tricyclic antidepressants, fentanyl, lithium, tramadol, St John's wort, and tryptophan can lead to a life-threatening condition called serotonin syndrome [5]. Diagnosis of serotonin syndrome is based on Hunter's criteria which require the usage of a serotonergic agent and one of the following: spontaneous clonus, inducible clonus plus agitation or diaphoresis, ocular clonus plus agitation or diaphoresis, tremor plus hyperreflexia, and hypertonia plus temperature above $38^{\circ} \mathrm{C}$ plus ocular clonus or inducible clonus [6]. Treatment consists of discontinuing serotonergic agents, supportive care, and administration of cyproheptadine.

PRES was first described as a separate neurological entity in 1996 [2]. PRES is increasingly becoming recognized as an interesting clinical, and radiological syndrome. In patients with PRES, symptoms in order of prevalence include altered mental status, headache, seizure, visual disturbances, focal neurological deficits, and status epileptics [1]. There are multiple known etiologies for PRES, with hypertension being the most common amongst them [2]. Other etiologies include eclampsia, systemic lupus erythematosus, autoimmune disorders, and immunosuppressive medications [3, 4, 7-9]. Cytotoxic medications such as cisplatin, cyclophosphamide, interferon, erythropoietin, tacrolimus, cyclosporine, and azathioprine can all induce PRES [1]. Drugs that antagonize the action of vascular endothelial growth factor such as bevacizumab, sunitinib, and sorafenib are also implicated in PRES [10-12].

PRES is diagnosed based on clinical and radiological assessment. Brain MRI, in particular T2-weighted sequences such as fluid-attenuated inversion recovery (FLAIR), is much more sensitive than CT head for diagnosis of PRES [13]. There are three main imaging patterns seen with PRES: a dominant parieto-occipital pattern, superior frontal sulcus pattern, and holohemispheric watershed pattern [13]. Treatment for PRES includes correcting the elevated blood pressure, removing the offending agent, starting anti-epileptic if seizures are present, and treating the underlying cause. The overall prognosis of PRES is good if diagnosed and treated early. Both the symptoms and imaging findings of PRES reverse after prompt discontinuation of the offending agent and appropriate treatment [7].

Although our patient had some other contributing factors that could have led to PRES, like hypertension and dialysis disequilibrium syndrome, after removal of the offending agents (venlafaxine and buspirone) our patient had prompt recovery. The most likely pathogenesis from SSRI usage seems to be the transient disturbance in the control of cerebrovascular tone. According to the proposed autoregulatory mechanism, the pressure-induced myogenic constriction of cerebral arteries by SSRI is one of the critical pathways along with cerebral microvascular circulation and calcium channel antagonist effects leading to smooth muscle calcium homeostasis [14-16]. Although RCVS and PRES have some overlapping features, with negative head and neck MRA it was excluded from the differential diagnosis, but RCVS has been reported in a patient presenting with serotonin syndrome [17].

We believe in our case, the use of venlafaxine with buspirone led to the development of serotonin syndrome that resulted in the patient having PRES. To our knowledge, this is one of the first cases of venlafaxine/buspirone causing PRES.

In conclusion, serotonin syndrome presenting with PRES is a rare phenomenon. However, it can occur in the setting of venlafaxine and buspirone usage as well. Knowledge of this interesting side effect is vital in diagnosis and treatment of PRES. Discontinuation of both agents along with supportive care will result in the resolution of symptoms and imaging findings. 
Malik et al.: Serotonin Syndrome Presenting as a Posterior Reversible Encephalopathy Syndrome

\section{Statement of Ethics}

Written informed consent was obtained from the patient.

\section{Disclosure Statement}

The authors have no conflicts of interest to declare.

\section{Funding Sources}

No funding was received for the publication of this case report.

\section{Author Contributions}

Muhammad Taimur Malik was the primary author. Critical editing and revision of the manuscript by Mohammad Faraz Majeed and Ramin Zand.

\section{References}

1 Fugate JE, Rabinstein AA. Posterior reversible encephalopathy syndrome: clinical and radiological manifestations, pathophysiology, and outstanding questions. Lancet Neurol. 2015 Sep;14(9):914-25.

2 Schwartz RB, Jones KM, Kalina P, Bajakian RL, Mantello MT, Garada B, et al. Hypertensive encephalopathy: findings on CT, MR imaging, and SPECT imaging in 14 cases. AJR Am J Roentgenol. 1992 Aug;159(2):379-83.

3 Hinchey J, Chaves C, Appignani B, Breen J, Pao L, Wang A, et al. A reversible posterior leukoencephalopathy syndrome. N Engl J Med 1996;334(8):494-500.

4 Raps EC, Galetta SL, Broderick M, Atlas SW. Delayed peripartum vasculopathy: cerebral eclampsia revisited. Ann Neurol. 1993 Feb;33(2):222-5.

5 Boyer EW, Shannon M. The serotonin syndrome. N Engl J Med. 2005 Mar;352(11):1112-20.

6 Dunkley EJ, Isbister GK, Sibbritt D, Dawson AH, Whyte IM. The Hunter Serotonin Toxicity Criteria: simple and accurate diagnostic decision rules for serotonin toxicity. QJM. 2003 Sep;96(9):635-42.

7 Edvardsson B. Venlafaxine as single therapy associated with hypertensive encephalopathy. Springerplus. 2015 Feb;4(1):97.

8 Kur JK, Esdaile JM. Posterior reversible encephalopathy syndrome-an underrecognized manifestation of systemic lupus erythematosus. J Rheumatol. 2006 Nov;33(11):2178-83.

9 Fugate JE, Claassen DO, Cloft HJ, Kallmes DF, Kozak OS, Rabinstein AA. Posterior reversible encephalopathy syndrome: associated clinical and radiologic findings. Mayo Clin Proc. 2010 May;85(5):427-32.

10 Seet RC, Rabinstein AA. Clinical features and outcomes of posterior reversible encephalopathy syndrome following bevacizumab treatment. QJM. 2012 Jan;105(1):69-75.

11 Tlemsani C, Mir O, Boudou-Rouquette P, Huillard O, Maley K, Ropert S, et al. Posterior reversible encephalopathy syndrome induced by anti-VEGF agents. Target Oncol. 2011 Dec;6(4):253-8.

12 Marra A, Vargas M, Striano P, Del Guercio L, Buonanno P, Servillo G. Posterior reversible encephalopathy syndrome: the endothelial hypotheses. Med Hypotheses. 2014 May;82(5):619-22.

13 Bartynski WS, Boardman JF. Distinct imaging patterns and lesion distribution in posterior reversible encephalopathy syndrome. AJNR Am J Neuroradiol. 2007 Aug;28(7):1320-7.

14 Toth P, Csiszar A, Tucsek Z, Sosnowska D, Gautam T, Koller A, et al. Role of 20-HETE, TRPC channels, and $\mathrm{BKCa}$ in dysregulation of pressure-induced $\mathrm{Ca} 2+$ signaling and myogenic constriction of cerebral arteries in aged hypertensive mice. Am J Physiol Heart Circ Physiol. 2013a Dec;305(12):H1698-708.

15 Springo Z, Toth P, Tarantini S, Ashpole NM, Tucsek Z, Sonntag WE, et al. Aging impairs myogenic adaptation to pulsatile pressure in mouse cerebral arteries. J Cereb Blood Flow Metab. 2015 Mar;35(4):527-30.

16 Ungvari Z, Pacher P, Koller A. Serotonin reuptake inhibitor fluoxetine decreases arteriolar myogenic tone by reducing smooth muscle [Ca2+]i. J Cardiovasc Pharmacol. 2000 Jun;35(6):849-54. 


\section{Case Reports in Neurology}

\begin{tabular}{l|l}
\hline Case Rep Neurol 2020;12:63-68 \\
\hline DOI: 10.1159/000505907 & $\begin{array}{l}\text { @ 2020 The Author(s). Published by S. Karger AG, Basel } \\
\text { www.karger.com/crn }\end{array}$ \\
\hline
\end{tabular}

Malik et al.: Serotonin Syndrome Presenting as a Posterior Reversible Encephalopathy Syndrome

17 Lee WJ, Yeon JY, Jo KI, Kim JS, Hong SC. Reversible Cerebral Vasoconstriction Syndrome and Posterior Reversible Encephalopathy Syndrome Presenting with Deep Intracerebral Hemorrhage in Young Women. J Cerebrovasc Endovasc Neurosurg. 2015 Sep;17(3):239-45.

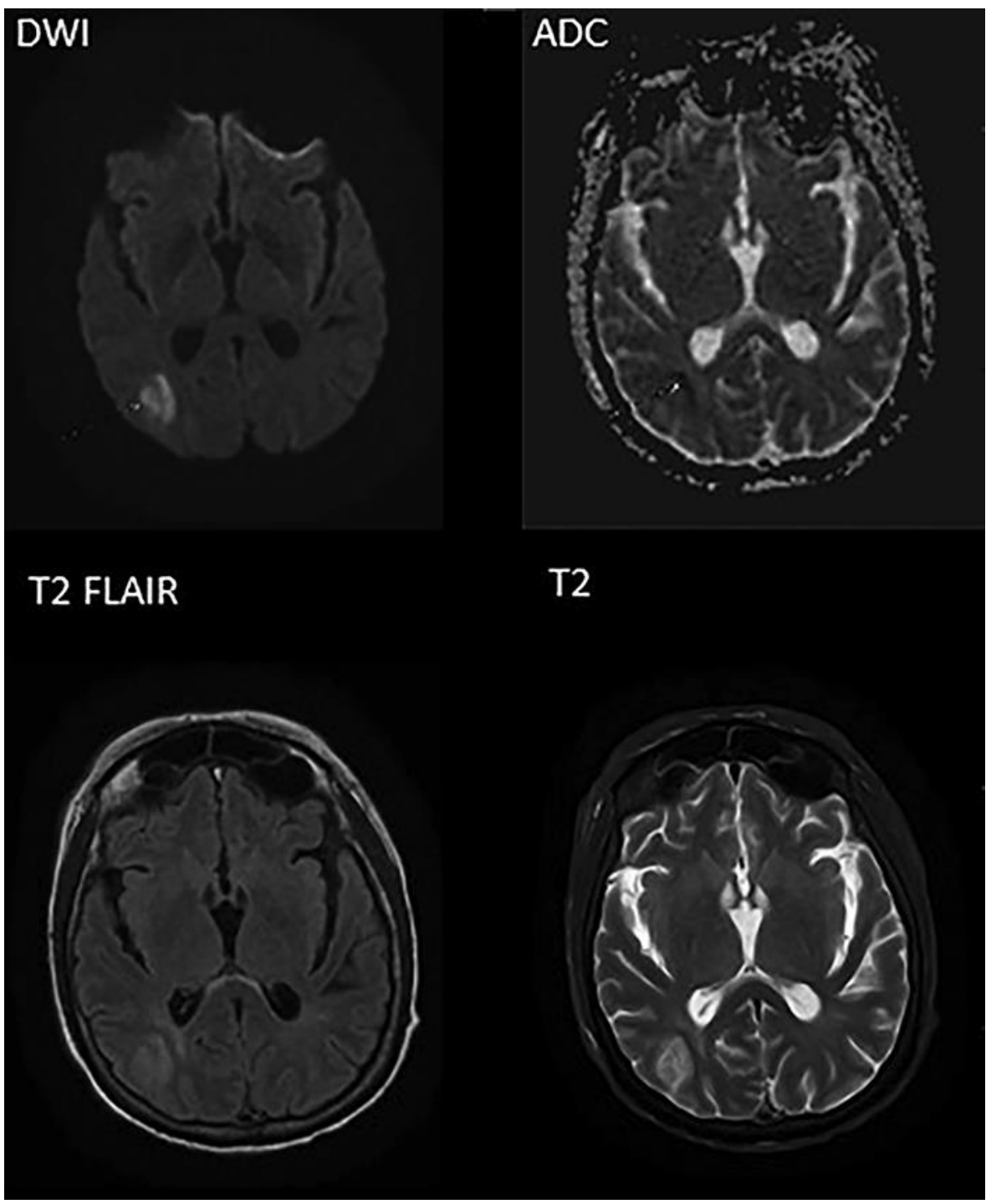

Fig. 1. MRI of the brain with and without contrast showing restricted diffusion in the right posterior temporal lobe and parietal lobes with associated T2/FLAIR hyperintensity. 


\section{Case Reports in Neurology}

\begin{tabular}{l|l} 
Case Rep Neurol 2020;12:63-68 \\
\hline DOI: 10.1159/000505907 & $\begin{array}{l}\text { (c) 2020 The Author(s). Published by S. Karger AG, Basel } \\
\text { www.karger.com/crn }\end{array}$
\end{tabular}

Malik et al.: Serotonin Syndrome Presenting as a Posterior Reversible Encephalopathy Syndrome

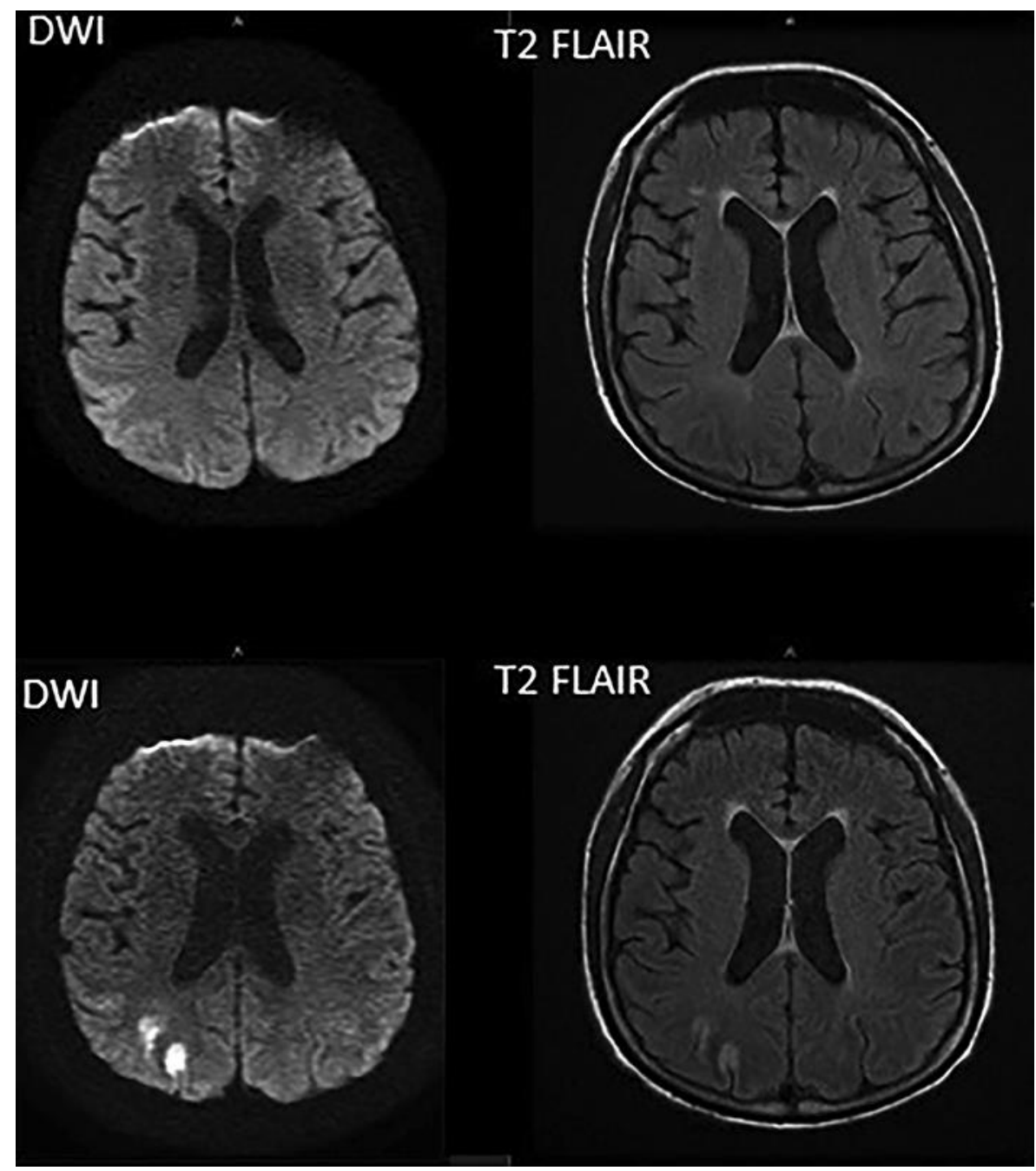

Fig. 2. Follow-up MRI of the brain with and without contrast 7 days later showing interval decrease in the restricted diffusion in the right posterior temporal lobe and parietal lobes with associated T2/FLAIR hyperintensity. 